\title{
Antihyperglycemic action of rhodiola-aqeous extract in type1-like diabetic rats
}

\author{
Chiang-Shan Niu* ${ }^{*}$, Li-Jen Chen ${ }^{2}$ and Ho-Shan Niu ${ }^{1}$
}

\begin{abstract}
Background: Rhodiola rosea (Rhodiola) is a plant in the Crassulaceae family that grows in cold regions of the world. It is mainly used in clinics as an adaptogen. Recently, it has been mentioned that Rhodiola increases plasma $\beta$-endorphin to lower blood pressure. Thus, the present study aims to investigate the antidiabetic action of Rhodiola in relation to opioids in streptozotocin-induced diabetic rats (STZ-diabetic rats).

Methods: In the present study, the plasma glucose was analyzed with glucose oxidase method, and the determination of plasma $\beta$-endorphin was carried out using a commercially available enzyme-linked immunosorbent assay. The adrenalectomy of STZ-diabetic rats was used to evaluate the role of $\beta$-endorphin. In addition, quantitative reverse transcription-polymerase chain reaction (qRT-PCR) and western blotting analysis were performed to investigate mRNA and protein expressions.
\end{abstract}

Results: Rhodiola-water extract dose-dependently lowered the plasma glucose in STZ-diabetic rats and this action was reversed by blockade of opioid $\mu$-receptors using cyprodime. An increase of plasma $\beta$-endorphin by rhodiola-water extract was also observed in same manner. The plasma glucose lowering action of rhodiola-water extract was attenuated in bilateral adrenalectomized rats. In addition, continuous administration of rhodiola-water extract for 3 days in STZ-diabetic rats resulted in an increased expression of glucose transporter subtype 4 (GLUT 4) in skeletal muscle and a marked reduction of phosphoenolpyruvate carboxykinase (PEPCK) expression in liver. These effects were also reversed by blockade of opioid $\mu$-receptors.

Conclusions: Taken together, rhodiola-water extract improves hyperglycemia via an increase of $\beta$-endorphin secretion from adrenal gland to activate opioid $\mu$-receptors in STZ-diabetic rats.

Keywords: Adrenalectomy, Hyperglycemia, Opioid $\mu$-receptor, Plasma glucose, Rhodiola-water extract

\section{Background}

Diabetes mainly results from dysfunction of glucose homeostasis and causes many complications, including cardiovascular diseases, neurological complication, chronic renal failure, and diabetic retinopathy [1-4]. Hyperglycemia is correlated with an increased risk of short-term acute complications, such as hyperosmolar coma, and long-term complications affecting the micro and macrovasculature $[5,6]$. In general, oral administration of antidiabetic agents, caloric restrictions, regular exercise and lifestyle are often applied to control hyperglycemia [7-9]. Recently, the management of diabetic hyperglycemia has attracted much attention in alternative therapy [10,11]. Moreover, herbal

\footnotetext{
* Correspondence: ncs838@yahoo.com.tw

'Department of Nursing, Tzu Chi College of Technology, Hualien City 97005, Taiwan

Full list of author information is available at the end of the article
}

supplements and other alternative medicine for handling of diabetic hyperglycemia are necessary.

Rhodiola rosea (Rhodiola) belongs to Crassulaceae family that is grown at high altitudes and northern latitudes and this herb is mentioned to reduce fatigue, improve physical activity and alleviate depression [12,13]. The documented hydrophilic extracts from Rhodiola contain many chemical compositions and they have been identified, including cinnamic alcohol, chlorogenic acid, rhodiooctanoside, rosiridin, rosavin and the phenolic compounds salidroside, rhodiolin in addition to a novel compound consisting of viridoside with an attached arabinose unit (mongrhoside) [13,14]. In recent years, rhodiola-water extract has been reported to improve hypertension via the release of $\beta$-endorphin in animal model [15]. Otherwise, involvement of $\beta$-endorphin or 
endogenous opioids in glucose lowering action of natural products has been mentioned [16,17]. Actually, some chemical agents or exogenous $\beta$-endorphin might improve hyperglycemia through an activation of opioid $\mu$-receptors in STZ-diabetic rats lacking insulin [18-20]. However, antihyperglycemic action of rhodiola-water extract remained obscure. In the present study, rhodiola-water extract is used to screen the effect on hyperglycemia in diabetic rats. Also, the mediation of $\beta$-endorphin in this action of rhodiola-water extract is further identified.

\section{Methods}

\section{Plant materials}

The radix of Rhodiola Rosea (Crassulaceae) originated from Qinghai-Tibet plateau of China was donated from Giu Ding Biotechnology Co., Ltd., Taiwan and was authenticated by Professor I-Min Liu (Department of Pharmacy, Tajen University). The dried voucher specimen (No. GiuDing 94001) was deposited in the herbarium of the college of pharmacy, Tajen University (Pingtung, Taiwan).

\section{Preparation of plant extracts}

The air-dried chopped plant radix (150 g) was extracted exhaustively by maceration with in distilled water $(1 \mathrm{~L})$ by stirring (Harmony Hot Plate Stirrer, Japan) at $55^{\circ} \mathrm{C}$ for $7 \mathrm{~h}$ (three times) and The extracts were sieved using a muslin cloth and then filtered under suction pressure with a filter paper. They were then concentrated under reduced pressure at $50^{\circ} \mathrm{C}$ using a rotary evaporator (Buchi, Switzerland). The crude water extract was then dried under vacuum at $50^{\circ} \mathrm{C}$ to yield the water-soluble fraction (WtF, $16.5 \mathrm{~g}$ ). The water-soluble fraction (WtF) was stored in a capped container and maintained at $4^{\circ} \mathrm{C}$. This product was obtained from Professor Shorong-Shii Liou (College of Pharmacy, Tajen University, Pingtung, Taiwan). The major active principles quantified in this product were salidroside $(8.4 \mathrm{mg} / \mathrm{g})$ and p-tyrosol $(1.9 \mathrm{mg} / \mathrm{g})$ as described in previous reports [21,22].

\section{Animal models}

Ten-week-old male Wistar rats weighing 250 to $300 \mathrm{~g}$ were obtained from the Animal Center of National Cheng Kung University Medical College. The diet of the animals used for the study was standard laboratory diet. The number of animals for each group of experiment is eight. STZ-diabetic rats were induced by intravenous injection (i.v.) of STZ (65 mg/kg) into Wistar rats according to the previous method [23]. Animals were considered to be type- 1 diabetes- like model if they showed plasma glucose concentrations of $20 \mathrm{mM}$ or greater in addition to polyuria and other diabetic features according to previous reports [24-30]. All studies were carried out 2 weeks after the injection of STZ. The rats used in the present study were maintained in accordance with the Guide for the Care and Use of Laboratory Animals of the National Institutes of Health, as well as the guidelines of the Animal Welfare Act and the study was approved by the animal research ethics committee of Tzu Chi College of Technology (TCCN-101006) which consisted of Prof. Yang SC, Chen MS, Dai KF, Liu WT, Wei TK, Cho LG, and Wu MY.

\section{Laboratory determinations}

The determination of plasma glucose was conducted according to the previous study [31]. The concentration of plasma glucose was measured by the glucose oxidase method using an analyzer (Quik-Lab, Ames; Miles Inc., Elkhart, IN, USA). The determination of BER in samples was carried out using a commercially available enzymelinked immunosorbent assay (Peninsula Laboratories, Belmont, CA, USA).

\section{Effect on plasma $\beta$-endorphin level in STZ-diabetic rats}

After fasting overnight, STZ-diabetic rats received an intraperitoneal treatment of rhodiola-water extract at the desired doses. It has been documented that rats receiving sodium pentobarbital showed no significant change in the parameters measured in plasma [32]. Thus, animals were anesthetized with sodium pentobarbital (35 mg/kg i.p.) and blood samples $(0.1 \mathrm{~mL})$ were coulected from the femoral vein for measurement of plasma glucose concentrations and $\beta$-endorphin (BER). In the present study, rhodiola-water extract at $75 \mathrm{mg} / \mathrm{kg}$ was found to produce the maximal plasma glucoselowering effect in STZ-diabetic rats $60 \mathrm{~min}$ after intraperitoneal injection. Thus, the effect of rhodiola-water extract on plasma BER was determined using blood samples collected at $60 \mathrm{~min}$ after the treatment. STZdiabetic rats that received an intraperitoneal treatment of vehicle only $(0.9 \%$ saline $)$ were used as controls. Further experiments were performed with the pretreatment of inhibitors, such as the antagonists of opioid $\mu$-receptors cyprodime $(1.0 \mathrm{mg} / \mathrm{kg})$ purchased from (Tocris Cookson, Bristol, UK). The antagonist was injected intravenously into rats $30 \mathrm{~min}$ before the treatment of rhodiola-water extract.

\section{Adrenalectomy of STZ-diabetic rats}

Bilateral adrenalectomy was performed using the dorsal approach under pentobarbital anesthesia (30 mg/kg, i.p.) as described previously [28]. Wistar rats to be adrenalectomized were fed standard rat chow and $0.9 \%$ sodium chloride in their drinking water ad libitum prior to surgery. Wistar rats to receive a sham operation (controls) were fed standard rat chow and water ad libitum prior to surgery. Animals were allowed to recover for 2 weeks 
after the operations. The animals appeared alert and in good health. Following recovery, diabetes was induced by an injection of STZ as described above. The effect of rhodiola-water extract at $75 \mathrm{mg} / \mathrm{kg}$ was determined using blood samples collected at $60 \mathrm{~min}$ after the treatment.

\section{Quantitative reverse transcription-polymerase chain reaction (qRT-PCR)}

Total RNA was extracted from liver and soleus muscle tissue samples using Trizol reagent (Invitrogen). Two microgram of total RNA was used for the reverse transcription reaction, along with Superscriptase II (Invitrogen), oligo-dT, and random primers. The web-based assay design software from the Universal Probe Library Assay Design Center was used to design the TaqMan primer pairs and to select the appropriate hybridization probes. The reactions were performed in $20 \mu \mathrm{L}$ of a mixture consisting of $13.4 \mu \mathrm{L}$ of PCR buffer, $0.2 \mu \mathrm{L}$ of each probe $(20 \mu \mathrm{mol} / \mathrm{L}), 4 \mu \mathrm{L}$ of LightCycler TaqMan (Roche Diagnostics $\mathrm{GmbH}$ ) and $2 \mu \mathrm{L}$ of template cDNA. A LightCycler Detection System (Roche Applied Science, Germany) was used for amplification and detection. The PCR reaction was carried out as follows: one cycle of $95^{\circ} \mathrm{C}$ for $10 \mathrm{~min}, 45$ cycles of $94^{\circ} \mathrm{C}$ for $10 \mathrm{~s}, 60^{\circ} \mathrm{C}$ for $20 \mathrm{~s}$, and $72^{\circ} \mathrm{C}$ for $1 \mathrm{~s}$. The crossing point for each amplification curve was determined using the second derivative maximum method. The concentration of each gene was calculated with the aid of the LightCycler software using the respective standard curve as reference. Relative gene expression was expressed as a ratio of the target gene concentration to the housekeeping gene 36B4 concentration. The sequences of primers were forward $5^{\prime}$-tttctgttggtatgcataatttgtaat-3' and reverse $5^{\prime}$-ccagtagaggaggtcaacaacc-3' for GLUT 4 and forward 5 '-gatgacattgcctggatgaa- 3 ' and reverse 5 '-acccgttttctgggttgatg-3' for PEPCK.

\section{Western blotting analysis}

Western blotting analysis was carried out as previously described [28] and quantification was obtained from three individual experiments. After homogenization of liver and soleus muscle using a glass/Teflon homogenizer, the homogenates $(50 \mu \mathrm{g})$ were separated by sodium dodecyl sulfate-polyacrylamide gel electrophoresis, and Western blot analysis was performed using either an anti-rat GLUT 4 antibody purchased from (Abcam, Cambridge, U.K) in soleus muscle or an antirat PEPCK antibody from (Santa Cruz Biotechnology, $\mathrm{CA}$ ) in liver. The blots were probed with a goat polyclonal actin antibody from (Millipore, Billerica, MA, USA) to ensure that the amount of protein loaded into each lane of the gel was constant. Blots were incubated with the appropriate peroxidase-conjugated secondary antibodies. After removal of the secondary antibodies, the blots were washed and developed using the ECLWestern blotting system. Densities of the obtained immunoblots at $45 \mathrm{KDa}$ for GLUT 4, $69.5 \mathrm{KDa}$ for PEPCK, and $43 \mathrm{KDa}$ for actin were quantified using laser densitometer.

\section{Statistical analysis}

The plasma glucose-lowering activity of rhodiola-water extract was calculated as the percentage decrease of the initial glucose value according to the formula: (Gi-Gt)/ $\mathrm{Gi} \times 100 \%$, where $\mathrm{Gi}$ is the initial glucose concentration and Gt is the plasma glucose concentration after treatment of rhodiola-water extract. Data are expressed as the mean \pm S.E.M. for the number $(n)$ of animals in the group as indicated in tables and figures. Differences among groups were analyzed by one-way ANOVA. The Dunnett range post hoc comparisons were used to determine the source of significant differences where appropriate. A $p$-value of 0.05 or less was considered statistically significant.

\section{Results}

\section{Effects of rhodiola-water extract on plasma glucose} concentration and plasma $\beta$-endorphin-like immunoreactivity (BER) level in STZ-diabetic rats

STZ-diabetic rats treated with rhodiola-water extract at $50 \mathrm{mg} / \mathrm{kg}$ through intraperitoneal injection produced the plasma glucose-lowering activities as $8.3 \pm 1.2 \%$ at $30 \mathrm{~min}$ later, $22.1 \pm 0.8 \%$ at $60 \mathrm{~min}$ later, $18.7 \pm 1.4 \%$ at $90 \mathrm{~min}$ later and $6.7 \pm 2.1 \%$ at $120 \mathrm{~min}$ later $(n=8)$. Then, we applied it at the most effective of time point in following experiments. After 60 minutes, rhodiola-water extract shows the plasma glucose-lowering activities as $11.6 \pm 1.1 \%, 21.1 \pm 0.9 \%, 32.9 \pm 3.2 \%$ in STZ-diabetic rats after an intraperitoneal (i.p.) injection at $35 \mathrm{mg} / \mathrm{kg}$, $50 \mathrm{mg} / \mathrm{kg}$ or $75 \mathrm{mg} / \mathrm{kg}$, respectively $(n=8)$. Rhodiolawater extract at $75 \mathrm{mg} / \mathrm{kg}$ significantly lowered the plasma glucose concentration from $381.4 \pm 5.7 \mathrm{mg} / \mathrm{dL}$ to $250.1 \pm 11.2 \mathrm{mg} / \mathrm{dL}(p<0.001 ; n=8)$ as shown in Figure 1A. Also, a dose-dependent increase of plasma BER level was observed in parallel in STZ-diabetic rats receiving rhodiola-water extract at same dosing from $35 \mathrm{mg} / \mathrm{kg}$ to $75 \mathrm{mg} / \mathrm{kg}$ (Figure 1B). Rhodiola-water extract at $75 \mathrm{mg} / \mathrm{kg}$ increased the plasma BER level from basal level of $66.0 \pm 0.9 \mathrm{pg} / \mathrm{mL}$ to $80.0 \pm 1.4 \mathrm{pg} / \mathrm{mL}$ in STZ-diabetic rats.

\section{Effect of opioid $\mu$-receptor blockade on rhodiola-water extract-induced plasma glucose lowering action in STZ-diabetic rats}

As shown in Figure 2, the inhibitory effect of cyprodime on the plasma glucose-lowering activity of rhodiolawater extract in STZ-diabetic rats was produced in a 

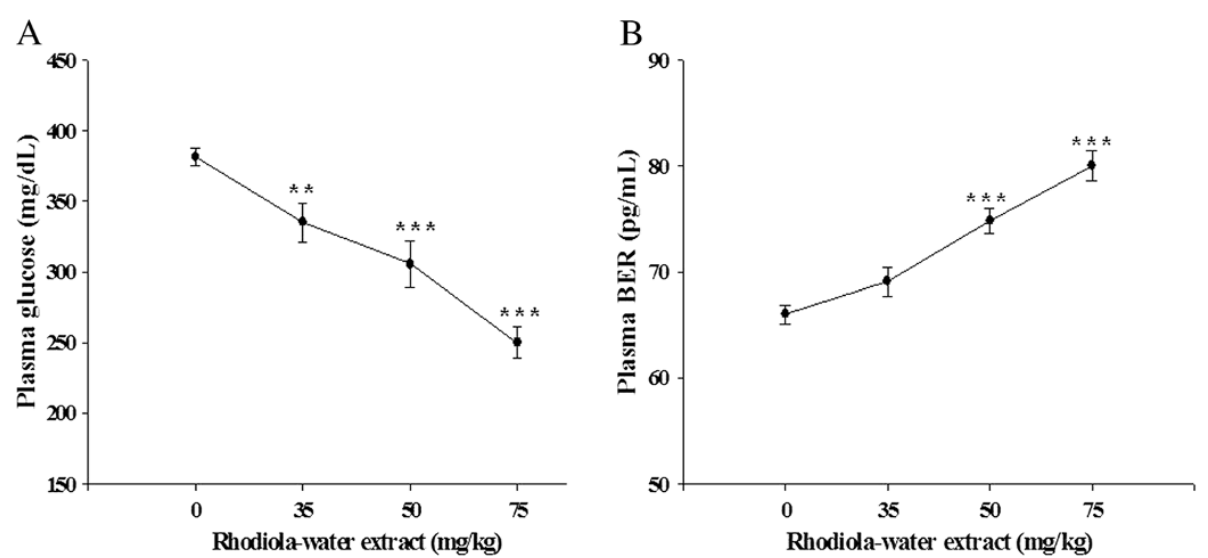

Figure 1 Effects of rhodiola-water extract on plasma glucose concentration and plasma $\beta$-endorphin level in STZ-diabetic rats. (A) The plasma glucose lowering activity produced by rhodiola-water extract through an intraperitoneal (i.p.) injection into STZ-diabetic rats. (B) Plasma $\beta$-endorphin-like immunoreactivity (BER) level in STZ-diabetic rats receiving i.p. injection of rhodiola-water extract. Values of mean and bar of S.E. M. were obtained from each group of 8 rats. Vehicle only ( $0.9 \%$ saline) was given at the same volume. ${ }^{* *} p<0.01$ and ${ }^{* * *}, p<0.001$ versus data from animals treated with vehicle (0).

dose-dependent manner. In the presence of $1 \mathrm{mg} / \mathrm{kg}$ cyprodime, the plasma glucose concentration in rats treated with $75 \mathrm{mg} / \mathrm{kg}$ rhodiola-water extract was $358.8 \pm 7.6 \mathrm{mg} / \mathrm{dL}$, which was not statistically different with the vehicle-treated STZ-diabetic rats (365.1 \pm $5.5 \mathrm{mg} / \mathrm{dL}$ ). However, cyprodime alone did not affect basal plasma glucose levels in STZ-diabetic rats.

Bilateral adrenalectomy of STZ-diabetic rats abolishes the effects of rhodiola-water extract on plasma glucose and BER levels

Bilateral adrenalectomy was performed in STZ-diabetic rats. Two weeks after adrenalectomy, there were no significant differences in the basal plasma levels of glucose and BER between sham-operated and adrenalectomized STZ-diabetic rats (Table 1). However, the actions of rhodiola-water extract regarding the lowering of plasma glucose and the elevation of plasma BER levels were abolished by bilateral adrenalectomy in STZ-diabetic rats while both were unchanged in the sham-operated STZ-diabetic rats (Table 1).

Effect of opioid $\mu$-receptor blockade on rhodiola-water extract-induced changes of GLUT 4 in skeletal muscle and hepatic PEPCK in STZ-diabetic rats

Treatment of STZ-diabetic rats with rhodiola-water extract $(75 \mathrm{mg} / \mathrm{kg})$ three times daily for 3 days resulted in an elevation of GLUT 4 expression level in skeletal

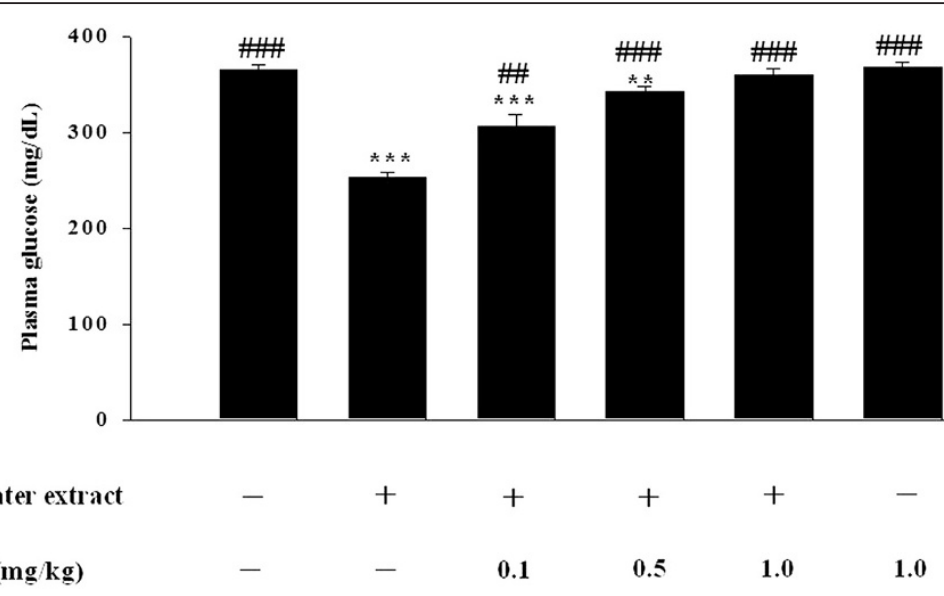

Figure 2 Effect of opioid $\mu$-receptor blockade on rhodiola-water extract-induced plasma glucose lowering action in STZ-diabetic rats. The antagonist was given by an intravenous (i.v.) injection at $30 \mathrm{~min}$ before the injection of rhodiola-water extract. Vehicle $(0.9 \% \mathrm{NaCl}$ in distilled water) was given at same volume. Values (mean \pm S.E.M.) were obtained from each group of 8 rats. ${ }^{* *}, p<0.01$ and ${ }^{* * *}, p<0.001$ compared with data from vehicle-treated STZ-diabetic rats. \#\#, $p<0.01$ and \#\#\#, $p<0.001$ compared with data from rhodiola-water extract (75 mg/kg)-treated STZ-diabetic rats. 
Table 1 Effect of adrenalectomy on the rhodiola-water extract-induced changes of $\beta$-endorphin-like immunoreactivity (BER) and plasma glucose concentration in STZ-diabetic rats

\begin{tabular}{lcc}
\hline & $\begin{array}{c}\text { Sham-operated } \\
\text { group }\end{array}$ & $\begin{array}{c}\text { Adrenalectomized } \\
\text { group }\end{array}$ \\
\hline Plasma glucose $(\mathrm{mg} / \mathrm{dL})$ & & \\
Basal & $378.6 \pm 5.3$ & $375.0 \pm 4.6$ \\
Vehicle & $370.5 \pm 6.9$ & $366.5 \pm 5.0$ \\
Rhodiola-water & $254.0 \pm 2.4^{* * *}$ & $373.5 \pm 5.8$ \\
extract $(75 \mathrm{mg} / \mathrm{kg})$ & & \\
Plasma BER $(\mathrm{pg} / \mathrm{mL})$ & & $69.8 \pm 1.2$ \\
Basal & $70.5 \pm 2.1$ & $69.0 \pm 1.4$ \\
Vehicle & $68.5 \pm 1.8$ & $68.7 \pm 1.2$ \\
Rhodiola-water & $84.3 \pm 1.3^{* * *}$ & \\
extract $(75 \mathrm{mg} / \mathrm{kg})$ & & \\
\hline
\end{tabular}

Blood samples from STZ-diabetic rats receiving an intraperitoneal injection of rhodiola-water extract or a vehicle-treated control were used for determination of $\beta$-endorphin-like immunoreactivity (BER) and the plasma glucose concentration. Vehicle $(0.9 \%$ saline) was given at the same volume. Values (mean \pm S.E. M.) were obtained from each group of eight rats. Basal level shows the value from fasted animals without treatment. ${ }^{* *} p<0.001$ versus basal value in each group.

muscle and this action was attenuated by cyprodime $(1.0 \mathrm{mg} / \mathrm{kg}$ ) (Figure 3A). In addition, the expression level of PEPCK in liver of STZ-diabetic rats was increased to about 2.2 folds of that in non-diabetic rats. The reduction of PEPCK expression by rhodiolawater extract $(75 \mathrm{mg} / \mathrm{kg})$ in diabetic rats was observed and this action was also attenuated by cyprodime (Figure 3B).
Effect of opioid $\mu$-receptor blockade on rhodiola-water extract-induced changes of protein levels of GLUT 4 in skeletal muscle and hepatic PEPCK in STZ-diabetic rats In Western blotting analysis, continuous treatment with rhodiola-water extract $(75 \mathrm{mg} / \mathrm{kg}$ ) increased the GLUT 4 protein level in skeletal muscle. Pretreatment of STZdiabetic rats with cyprodime $(1.0 \mathrm{mg} / \mathrm{kg})$ abolished this action of rhodiola-water extract (Figure 4A). Similarly, the protein level of PEPCK in liver of STZ-diabetic rats was raised to approximately 1.8 folds of that in non-diabetic rats. The protein level of hepatic PEPCK in diabetic rats was reversed by similar treatment with rhodiola-water extract to normal level and this action was also abolished by cyprodime (Figure 4B). The data for quantification of GLUT 4 and PEPCK protein levels were indicated in Figure $4 \mathrm{~A}$ and Figure $4 B$, respectively.

\section{Discussion}

In the present study, rhodiola-water extract lowers plasma glucose concurrently with an increase of plasma BER in STZ-diabetic rats. Both effects of rhodiola-water extract are dose-dependent in same dose ranges. STZdiabetic rats are widely used as type-1 like diabetic animal model to investigate hyperglycemia [33]. Therefore, the plasma glucose-lowering and BER-elevating actions of rhodiola-water extract are concluded to occur under the lacking of insulin. When treated with rhodiola-water extract, STZ-diabetic rats provide a suitable model to explore the phenomena that the reduction of plasma glucose concentrations and increased BER are associated in diabetic rats.
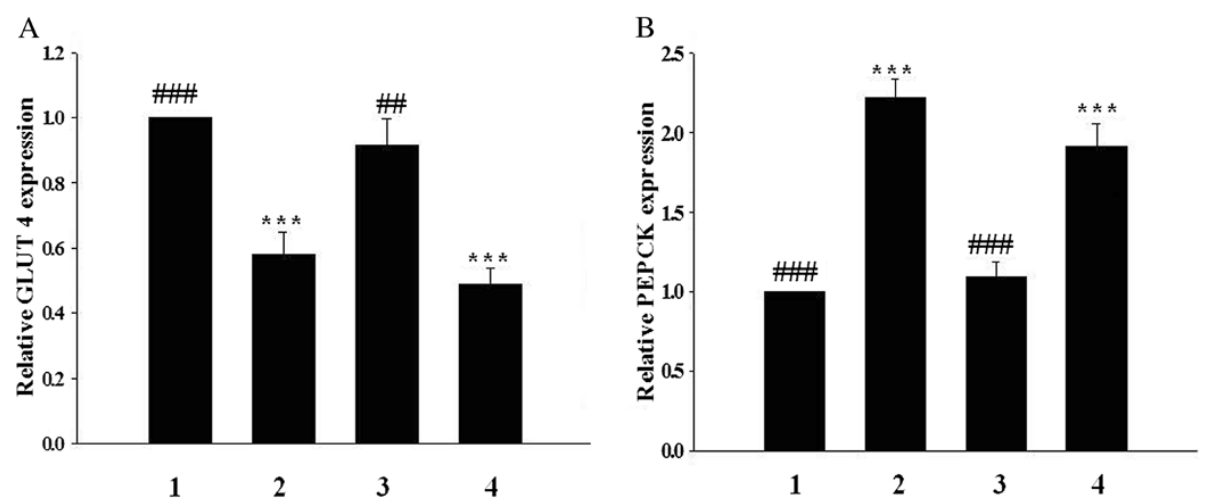

Figure 3 Effect of opioid $\mu$-receptor blockade on rhodiola-water extract-induced changes of GLUT 4 in skeletal muscle and hepatic PEPCK in STZ-diabetic rats. (A) The mRNA level of GLUT 4 in skeletal muscle isolated from STZ-diabetic rats receiving treatment with rhodiola-water extract only or with cyprodime in combination, three times daily for 3 days. (B) The mRNA level of PEPCK expression in liver isolated from STZ-diabetic rats receiving same treatment with rhodiola-water extract only or with cyprodime in combination. The samples were then collected for qRT-PCR analysis. Lane1, vehicle-treated Wistar rats; lane2, vehicle-treated STZ-diabetic rats; lane 3, rhodiola-water extract (75 mg/kg)-treated STZ-diabetic rats; lane 4, rhodiola-water extract $(75 \mathrm{mg} / \mathrm{kg}$ ) plus cyprodime $(1.0 \mathrm{mg} / \mathrm{kg}$ )-treated STZ-diabetic rats. Data are expressed as mean with standard error (SE) ( $n=6$ per group) is indicated in each column. ${ }^{* *}, p<0.001$ compared with data obtained from lane 1. \#\#, $p<0.01$ and \#\#, $p<0.001$ compared with data obtained from lane 2. 

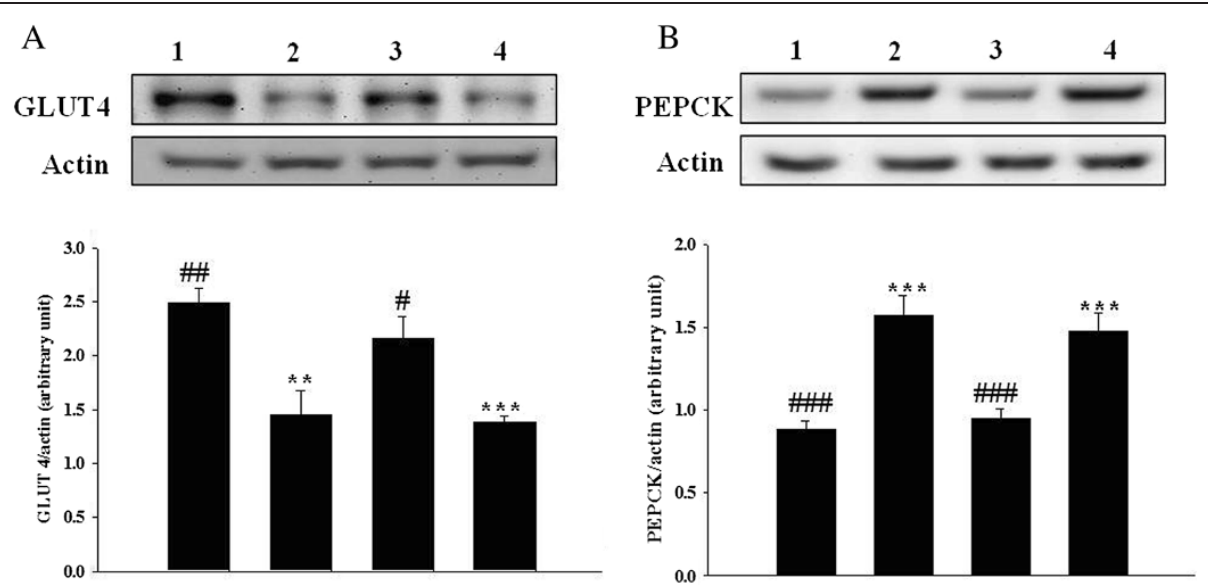

Figure 4 Effect of opioid $\mu$-receptor blockade on rhodiola-water extract-induced changes of protein levels of GLUT 4 in skeletal muscle and hepatic PEPCK in STZ-diabetic rats. (A) The representative response of protein level for GLUT 4 or actin in skeletal muscle isolated from STZ-diabetic rats receiving treatment with rhodiola-water extract only or with cyprodime in combination, three times daily for 3 days. (B) The representative response of protein level for PEPCK or actin in liver isolated from STZ-diabetic rats receiving same treatment with rhodiola-water extract only or with cyprodime in combination. Lane1, vehicle-treated Wistar rats; lane2, vehicle-treated STZ-diabetic rats; lane 3, rhodiola-water extract (75 mg/kg)-treated STZ-diabetic rats; lane 4, rhodiola-water extract (75 mg/kg) plus cyprodime $(1.0 \mathrm{mg} / \mathrm{kg})$-treated STZ-diabetic rats. Quantification of protein level using GLUT 4/actin or PEPCK/actin showing mean with standard error (SE) ( $n=6$ per group) in each column is indicated in the lower panel. ${ }^{* *}, p<0.01$ and ${ }^{* * *}, p<0.001$ compared with data obtained from lane 1. \#, $p<0.05, \# \#, p<0.01$ and \#\#\#, $p<0.001$ compared with data obtained from lane 2 .

Many actions of endogenous $\beta$-endorphin are recognized to be mediated by the opioid $\mu$-receptors including the regulation of glucose homeostasis [34,35], but the mediation of opioid delta-receptor located in skeletal muscle regarding to the hypoglycemic effect of BER has also been demonstrated [36,37]. Actually, it has been indicated that the expression of $\beta$-endorphin sensitive receptor on skeletal muscle is vastly increased in type 1 and type 2 diabetic animals [38,39]. Moreover, the increase of hepatic opioid $\mu$-receptors was related to diabetic hyperglycemia [40]. Thus, opioid $\mu$-receptor blockade was employed to evaluate the involvement of this receptor in rhodiola-water extract-induced plasma glucose lowering action in STZ-diabetic rats. The blood glucose lowering effect of rhodiola-water extract is similar to meforminin in STZ-diabetic rats as described in previous reports [41,42]. Cyprodime, the selective opioid $\mu$-receptor antagonist, has been shown to effectively abolish the activation of opioid $\mu$-receptors $[43,44]$. The ability of rhodiola-water extract to decrease plasma glucose was actually suppressed by blockade of opioid $\mu$-receptors with cyprodime. These findings demonstrate the activation of opioid $\mu$-receptors through increased circulating $\beta$-endorphin in the plasma glucose lowering action of rhodiola-water extract in diabetic rats. Similar action was also observed in another herbal principle [45].

Endogenous opioids can be released into the bloodstream from glands other than the pituitary gland [46], and the secretion of opioids from the adrenal gland was observed to foster a reduction in plasma glucose in STZ- diabetic rats [28]. Thus, bilateral adrenalectomy was performed in the present study to verify that the source of the increased plasma BER observed in STZ-diabetic rats in response to rhodiola-water extract was the adrenal gland. As shown in Table 1, the plasma glucose lowering action and the increase of BER induced by rhodiola-water extract in STZ-diabetic rats were both attenuated by adrenalectomy. Thus, secretion of endogenous $\beta$-endorphin from adrenal gland is responsible for the plasma glucose lowering action of rhodiola-water extract in STZ-diabetic rats. Decrease of plasma glucose by $\beta$-endorphin has been mentioned in previous reports $[47,48]$.

In diabetes, hyperglycemia is considered the consequence of increased hepatic glucose output and decreased peripheral glucose uptake [49,50]. Insulin deficiency is associated with changes in hepatic metabolism including increased expression of PEPCK that is a key enzyme of hepatic carbohydrate metabolism [49,51]. Additionally, decreased expression of skeletal muscle GLUT 4 in diabetes resulted in the reduction of insulin-mediated glucose uptake into skeletal muscle [52]. It was interesting to ascertain whether rhodiola-water extract produced plasma glucose lowering action in diabetic rats by overturning the diabetes dependent reduction of GLUT 4 expression and/ or increase in PEPCK expression. To provide sample time for alterations in gene expression, STZ-diabetic rats received the repeated treatment rhodiola-water extract for 3 days. Under these conditions, the increase in hepatic PEPCK gene expression due to hyperglycemia was abolished by rhodiola-water extract. The decrease of GLUT 4 
expression in skeletal muscle was also reversed by the same repeated treatment rhodiola-water extract. These findings suggest that rhodiola-water extract shows plasma glucose lowering action due to changes in the expressions of hepatic PEPCK and muscle GLUT 4 in an insulinindependent manner.

It has been indicated that endogenous $\beta$-endorphin via activation of opioid $\mu$-receptors located in peripheral tissues was found to serve as a positive regulator of glucose utilization and a negative modulator of hepatic gluconeogenesis in the insulin-deficient state [20,53]. Opioid $\mu$-receptors antagonist was therefore used to evaluate the involvement of opioid $\mu$-receptors in the effects of rhodiola-water extract on metabolic gene expression in diabetic rats. Actions of rhodiola-water extract regarding the increase of GLUT 4 expression in skeletal muscle and decrease of PEPCK expression in liver were abolished in STZ-diabetic rats with the blockade of opioid $\mu$-receptors using antagonist (Figure 3). Furthermore, elevation of GLUT 4 protein expression and suppression of PEPCK protein expression in STZ-diabetic rats by rhodiola-water extract were also blocked by the same antagonist named cyprodime (Figure 4).

It has been reported that $\mathrm{PKC}$ is involved in the ratelimiting step in GLUT4 mRNA expression [54,55]. Also, the PLC-PKC pathway is mediated in opioid $\mu$-receptor activation [20,44]. Thus, this pathway is involved in the regulation of GLUT4 gene expression, although the detailed mechanism(s) need more investigations in the future. Our data also consistent with the report showing that opioid $\mu$-receptor activation might act as a negative regulator to modify the gene expression of PEPCK in insulin-deficient state $[19,56]$. However, the mediation of PLC-PKC pathway in regulation of PEPCK gene expression is still unclear [57]. Thus, the signals from opioid $\mu$-receptor activation for regulation of hepatic PEPCK gene expression during the absence of insulin need to clarify in the future. Taken together, the normalizations of hepatic PEPCK and muscle GLUT 4 expressions in STZ-diabetic rats by rhodiola-water extract are mainly mediated via an activation of opioid $\mu$-receptors mainly through the released endogenous $\beta$-endorphin.

Many ingredients are mentioned in rhodiola [58] including glycosides, flavonoids, phenilpropanoids, and others [59]. Also, another plant of the same genus showed merit in reduction of plasma glucose in type- 2 diabetic animals [60]. Thus, it is important to find out the effective ingredients and/or related metabolites in this action of rhodiola in the future.

\section{Conclusions}

In conclusion, our results suggest that rhodiola-water extract may enhance the secretion of endogenous $\beta$-endorphin from adrenal gland of STZ-diabetic rats.
The plasma glucose lowering action of rhodiola-water extract is mainly mediated by $\beta$-endorphin release through an activation of opioid $\mu$-receptors to achieve the higher of GLUT 4 gene expression and/or the decrease of hepatic PEPCK gene expression. Therefore, these findings provide evidence in support of rhodiolawater extract as the agent for handling of hyperglycemia in diabetic disorders in rats. However, the blood glucose lowering effect of rhodiola and/or active ingredient(s) on human needs more investigations in the future.

\section{Competing interests}

The authors declare that they have no competing interests.

\section{Authors' contributions}

CSN participated in the design of the study and drafted the manuscript. LJC carried out animal experiments, Western blotting analysis, GRT-PCR and performed the statistical analysis. HSN conceived of the study, and participated in its design and coordination and helped to analysis of statistical difference. All authors have read and approved the final manuscript.

\section{Acknowledgements}

We gratefully acknowledge the financial support by a grant (TCCT-1011A09) from Tzu Chi College of technology, Hualien City, Taiwan, R.O.C. We also thank Mr. Y.C. Chen for his skillful technical assistance.

\section{Author details}

'Department of Nursing, Tzu Chi College of Technology, Hualien City 97005, Taiwan. ${ }^{2}$ Institute of Basic Medical Sciences, College of Medicine, National Cheng Kung University, Tainan City 70101, Taiwan.

Received: 8 September 2013 Accepted: 9 January 2014

Published: 13 January 2014

\section{References}

1. Nanchen D, Rodondi N, Cornuz J, Hillier T, Ensrud KE, Cauley JA, Bauer DC: Mortality associated with diabetes and cardiovascular disease in older women. PLoS One 2012, 7:e48818.

2. Kumar B, Gupta SK, Saxena R, Srivastava S: Current trends in the pharmacotherapy of diabetic retinopathy. J Postgrad Med 2012, 58:132-139.

3. Brosius FC 3rd, Alpers CE: New targets for treatment of diabetic nephropathy: what we have learned from animal models. Curr Opin Nephrol Hypertens 2013, 22:17-25.

4. Kan M, Guo G, Singh B, Singh V, Zochodne DW: Glucagon-like peptide 1, insulin, sensory neurons, and diabetic neuropathy. J Neuropathol Exp Neurol 2012, 71:494-510.

5. Haak T: Initial combination with linagliptin and metformin in newly diagnosed type 2 diabetes and severe hyperglycemia. Adv Ther 2012, 29(12):1005-1015. doi: 10.1007/s12325-012-0066-0. Epub 2012 Nov 29.

6. Pugliese A: The multiple origins of type 1 diabetes. Diabet Med 2012. doi:10.1038/ng1708.

7. Hamdy O, Goodyear LJ, Horton ES: Diet and exercise in type 2 diabetes mellitus. Endocrinol Metab Clin North Am 2001, 30:883-907.

8. Onat A: Metabolic syndrome: nature, therapeutic solutions and options. Expert Opin Pharmacother 2011, 12:1887-1900.

9. Vinik Al, Cincotta AH, Scranton RE, Bohannon N, Ezrokhi M, Gaziano JM: Effect of bromocriptine-QR on glycemic control in subjects with uncontrolled hyperglycemia on one or two oral anti-diabetes agents. Endocr Pract 2012, 18(6):931-943. doi: 10.4158/EP12187.OR.

10. Yoo KM, Lee C, Lo YM, Moon B: The hypoglycemic effects of American red ginseng (Panax quinquefolius L.) on a diabetic mouse model. J Food Sci 2012, 77:H147-H152.

11. Pinto Mda S, Ranilla LG, Apostolidis E, Lajolo FM, Genovese MI, Shetty K: Evaluation of antihyperglycemia and antihypertension potential of native Peruvian fruits using in vitro models. J Med Food 2009, 12:278-291.

12. Chan SW: Panax ginseng, Rhodiola rosea and Schisandra chinensis. Int J Food Sci Nutr 2012, 63(1):75-81. 
13. Panossian A, Wikman G, Sarris J: Rosenroot (Rhodiola rosea): traditional use, chemical composition, pharmacology and clinical efficacy. Phytomedicine 2010, 17:481-493.

14. Wiedenfeld H, Dumaa M, Malinowski M, Furmanowa M, Narantuya S: Phytochemical and analytical studies of extracts from Rhodiola rosea and Rhodiola quadrifida. Pharmazie 2007, 62:308-311.

15. Lee WJ, Chung HH, Cheng YZ, Lin HJ, Cheng JT: Rhodiola-water extract induces beta-endorphin secretion to lower blood pressure in spontaneously hypertensive rats. Phytother Res 2012. doi: 10.1002/ptr.4900.

16. Liu IM, Cheng JT: Mediation of endogenous beta-endorphin in the plasma glucose-lowering action of herbal products observed in type 1-like diabetic rats. Evid Based Complement Altern Med 2011, 2011:987876.

17. Su CH, Liu IM, Chung HH, Cheng JT: Activation of I2-imidazoline receptors by agmatine improved insulin sensitivity through two mechanisms in type-2 diabetic rats. Neurosci Lett 2009, 457:125-128.

18. Liu IM, Chi TC, Chen YC, Lu FH, Cheng JT: Activation of opioid mu-receptor by loperamide to lower plasma glucose in streptozotocininduced diabetic rats. Neurosci Lett 1999, 265:183-186.

19. Cheng JT, Liu IM, Chi TC, Tzeng TF, Lu FH, Chang CJ: Plasma glucoselowering effect of tramadol in streptozotocin-induced diabetic rats. Diabetes 2001, 50:2815-2821.

20. Cheng JT, Liu IM, Tzeng TF, Tsai CC, Lai TY: Plasma glucose-lowering effect of beta-endorphin in streptozotocin-induced diabetic rats. Horm Metab Res 2002, 34:570-576.

21. Ming DS, Hillhouse BJ, Guns ES, Eberding A, Xie S, Vimalanathan S, Towers GH: Bioactive compounds from Rhodiola rosea (Crassulaceae). Phytother Res 2005, 19:740-743.

22. Guo N, Hu Z, Fan X, Zheng J, Zhang D, Xu T, Yu T, Wang Y, Li H: Simultaneous determination of salidroside and its aglycone metabolite p-tyrosol in rat plasma by liquid chromatography-tandem mass spectrometry. Molecules 2012, 17:4733-4754

23. Liu IM, Chen WC, Cheng JT: Mediation of beta-endorphin by isoferulic acid to lower plasma glucose in streptozotocin-induced diabetic rats. J Pharmacol Exp Ther 2003, 307:1196-1204.

24. Luippold G, Klein T, Mark M, Grempler R: Empagliflozin, a novel potent and selective SGLT-2 inhibitor, improves glycaemic control alone and in combination with insulin in streptozotocin-induced diabetic rats, a model of type 1 diabetes mellitus. Diabetes Obes Metab 2012, 14:601-607.

25. Moreira-Rodrigues M, Quelhas-Santos J, Serrao P, Fernandes-Cerqueira C, Sampaio-Maia B, Pestana M: Glycaemic control with insulin prevents the reduced renal dopamine D1 receptor expression and function in streptozotocin-induced diabetes. Nephrol Dial Transplant 2010, 25:2945-2953.

26. Yu BC, Chang CK, Su CF, Cheng JT: Mediation of beta-endorphin in andrographolide-induced plasma glucose-lowering action in type I diabetes-like animals. Naunyn Schmiedebergs Arch Pharmacol 2008, 377:529-540.

27. Niu HS, Hsu FL, Liu IM, Cheng JT: Increase of beta-endorphin secretion by syringin, an active principle of eleutherococcus senticosus, to produce antihyperglycemic action in type 1-like diabetic rats. Horm Metab Res 2007, 39:894-898.

28. Hwang SL, Liu IM, Tzeng TF, Cheng JT: Activation of imidazoline receptors in adrenal gland to lower plasma glucose in streptozotocin-induced diabetic rats. Diabetologia 2005, 48:767-775

29. Chan P, Wong KL, Liu IM, Tzeng TF, Yang TL, Cheng JT: Antihyperglycemic action of angiotensin II receptor antagonist, valsartan, in streptozotocininduced diabetic rats. J Hypertens 2003, 21:761-769.

30. Cheng JT, Liu IM, Chi TC, Tzeng TF: Release of beta-endorphin by prostaglandin E2 to lower plasma glucose in streptozotocin-induced diabetic rats. Horm Metab Res 2001, 33:439-443.

31. Huang $\mathrm{CH}$, Chen MF, Chung HH, Cheng JT: Antihyperglycemic effect of syringaldehyde in streptozotocin-induced diabetic rats. J Nat Prod 2012, 75:1465-1468.

32. Johansen $\mathrm{O}$, Vaaler $\mathrm{S}$, Jorde $\mathrm{R}$, Reikeras $\mathrm{O}$ : Increased plasma glucose levels after hypnorm anaesthesia, but not after pentobarbital anaesthesia in rats. Lab Anim 1994, 28:244-248.

33. Biswas M, Kar B, Bhattacharya S, Kumar RB, Ghosh AK, Haldar PK: Antihyperglycemic activity and antioxidant role of terminalia arjuna leaf in streptozotocin-induced diabetic rats. Pharm Biol 2011, 49:335-340.

34. Zeng L, Wan L, Chen L, Li S, Lu Y, Huang Q, Wang L, Li Y, Cheng J, Lu X: Selective depletion of activated $T$ cells by recombinant immunotoxin containing anti-CTLA-4 single-chain fragment of variable antibody and N-terminal fragment of perforin. Transplant Proc 2006, 38:2151-2153.

35. Niu HS, Hsu FL, Liu IM, Cheng JT: Increase of beta-endorphin secretion by syringin, an active principle of eleutherococcus senticosus, to produce antihyperglycemic action in type 1-like diabetic rats. Horm Metab Res 2007, 39:894-898.

36. Evans AA, Khan S, Smith ME: Evidence for a hormonal action of beta-endorphin to increase glucose uptake in resting and contracting skeletal muscle. J Endocrinol 1997, 155:387-392.

37. Evans AA, Tunnicliffe G, Knights P, Bailey CJ, Smith ME: Delta opioid receptors mediate glucose uptake in skeletal muscles of lean and obese-diabetic (ob/ob) mice. Metabolism 2001, 50:1402-1408.

38. Hughes S, Smith ME, Bailey CJ: Beta-endorphin and corticotropin immunoreactivity and specific binding in the neuromuscular system of obese-diabetic mice. Neuroscience 1992, 48:463-468.

39. Hughes S, Smith ME, Bailey CJ: POMC-derived peptides in the neuromuscular system of streptozotocin-diabetic mice. Peptides 1992, 13:873-877.

40. Cheng JT, Liu IM, Chi TC, Tzeng TF: Increase of opioid mu-receptor gene expression in streptozotocin-induced diabetic rats. Horm Metab Res 2001, 33:467-471.

41. Cheng JT, Huang CC, Liu IM, Tzeng TF, Chang CJ: Novel mechanism for plasma glucose-lowering action of metformin in streptozotocin-induced diabetic rats. Diabetes 2006, 55:819-825.

42. Tzeng TF, Liou SS, Chang CJ, Liu IM: The ethanol extract of zingiber zerumbet attenuates streptozotocin-induced diabetic nephropathy in rats. Evid Based Complement Altern Med 2013, 2013:340645

43. Chen ZC, Shieh JP, Chung HH, Hung CH, Lin HJ, Cheng J: Activation of peripheral opioid micro-receptors in blood vessel may lower blood pressure in spontaneously hypertensive rats. Pharmacology 2011, 87:257-264.

44. Lee LM, Lin CS, Chung HH, Lin KC, Cheng JT: Urinary bladder relaxation through activation of opioid mu-receptors induced by loperamide is increased in diabetic rats. Exp Clin Endocrinol Diabetes 2012, 120:323-328.

45. Niu CS, Chen W, Wu HT, Cheng KC, Wen YJ, Lin KC, Cheng JT: Decrease of plasma glucose by allantoin, an active principle of yam (Dioscorea spp.), in streptozotocin-induced diabetic rats. J Agric Food Chem 2010, 58:12031-12035.

46. Guillemin R, Vargo T, Rossier J, Minick S, Ling N, Rivier C, Vale W, Bloom F: Beta-endorphin and adrenocorticotropin are selected concomitantly by the pituitary gland. Science 1977, 197:1367-1369.

47. Lai DM, Tu YK, Liu IM, Chen PF, Cheng JT: Mediation of beta-endorphin by ginsenoside Rh2 to lower plasma glucose in streptozotocin-induced diabetic rats. Planta Med 2006, 72:9-13.

48. Liu IM, Liou SS, Cheng JT: Mediation of beta-endorphin by myricetin to lower plasma glucose in streptozotocin-induced diabetic rats. J Ethnopharmacol 2006, 104:199-206.

49. Consoli A, Nurjhan N, Capani F, Gerich J: Predominant role of gluconeogenesis in increased hepatic glucose production in NIDDM. Diabetes 1989, 38:550-557.

50. Wi JK, Kim JK, Youn JH: Mechanisms of postabsorptive hyperglycemia in streptozotocin diabetic rats. Am J Physiol 1996, 270:E752-E758.

51. Gomez-Valades AG, Vidal-Alabro A, Molas M, Boada J, Bermudez J, Bartrons $R$, Perales JC: Overcoming diabetes-induced hyperglycemia through inhibition of hepatic phosphoenolpyruvate carboxykinase (GTP) with RNAi. Mol Ther 2006, 13:401-410.

52. Leto D, Saltiel AR: Regulation of glucose transport by insulin: traffic control of GLUT4. Nat Rev Mol Cell Biol 2012, 13:383-396.

53. Shieh JP, Cheng KC, Chung HH, Kerh YF, Yeh CH, Cheng JT: Plasma glucose lowering mechanisms of catalpol, an active principle from roots of Rehmannia glutinosa, in streptozotocin-induced diabetic rats. J Agric Food Chem 2011, 59:3747-3753.

54. Seatter MJ, Gould GW: The mammalian facilitative glucose transporter (GLUT) family. Pharm Biotechnol 1999, 12:201-228.

55. Pessin JE, Bell Gl: Mammalian facilitative glucose transporter family: structure and molecular regulation. Annu Rev Physiol 1992, 54:911-930.

56. Cheng JT, Liu IM, Tzeng TF, Tsai CC, Lai TY: Plasma glucose-lowering effect of beta-endorphin in streptozotocin-induced diabetic rats. Horm Metab Res 2002, 34:570-576.

57. Kai Y, Matsumura H, Izui K: Phosphoenolpyruvate carboxylase: three-dimensional structure and molecular mechanisms. Arch Biochem Biophys 2003, 414:170-179. 
58. Panossian A, Wikman G, Sarris J: Rosenroot (Rhodiola rosea): traditional use, chemical composition, pharmacology and clinical efficacy. Phytomedicine 2010, 17:481-493.

59. Peschel W, Prieto JM, Karkour C, Williamson EM: Effect of provenance, plant part and processing on extract profiles from cultivated European Rhodiola rosea L. for medicinal use. Phytochemistry 2013, 86:92-102.

60. Wang J, Rong X, Li W, Yang Y, Yamahara J, Li Y: Rhodiola crenulata root ameliorates derangements of glucose and lipid metabolism in a rat model of the metabolic syndrome and type 2 diabetes. J Ethnopharmacol 2012, 142:782-788.

doi:10.1186/1472-6882-14-20

Cite this article as: Niu et al: Antihyperglycemic action of rhodiolaaqeous extract in type1-like diabetic rats. BMC Complementary and Alternative Medicine 2014 14:20.

\section{Submit your next manuscript to BioMed Central and take full advantage of:}

- Convenient online submission

- Thorough peer review

- No space constraints or color figure charges

- Immediate publication on acceptance

- Inclusion in PubMed, CAS, Scopus and Google Scholar

- Research which is freely available for redistribution 